\title{
Effectual Routine for Trilateral Authentication in Ad-hoc Networks using Multicast Conventions
}

\author{
S. Anjanaa ${ }^{1,}$ M. Nathiya ${ }^{2,}$ T. Shampavi' ${ }^{3,}$ M. Sujitha ${ }^{4}$ \\ Department of Computer Science and Engineering Shivani Engineering College, Trichy, India
}

\begin{abstract}
Ad-hoc networks have mass of applications; though, a dynamic problem regarding their security aspects must be answered in order to realize these applications. Validating the source and ensuring the integrity of the message traffic become an essential constraint for the operation and management of the network. We focus on the security problem of protecting the network connectivity between nodes in ad-hoc network. New nodes start to contribute in the network as guests; they can only become full members with a network signed certificate after their authenticity has been warranted by some other members. In this paper we introduce routine of multicast Protocol for tired authentication between the multilevel of ad-hoc network. In this proposed protocol Central Authority with trilateral authentication for managing key authentication and certification process which leads to increase the security and reliability of the network.
\end{abstract}

Keywords---Central Authority, Multicast Security, Wireless Network, Tiered Network, WANET.

\section{Introduction}

Topology changes, bandwidth limitations and energy restriction pose various troubles in the absence of a fixed infrastructure in hybrid and self-motivated ad-hoc wireless networks. Securing such WANETs is a particularly difficult task because of the vulnerability of the wireless "links", reduced physical protection of the nodes, the periodic nature of connectivity and the dynamically varying topology. Generally, multicast traffic among the nodes has to be delivered in a secure and trusted manner. The network services requisite to accomplish these three security goals: (1) Confidentiality, to avoid antagonists from reading communicated data. (2) Message integrity, to prevent modifying transmitted messages. (3) Source Authentication, to avert man-inthe-middle attacks that replay transmitted data for node impersonation.

\section{A. Security in Ad-Hoc Network}

Ad-hoc networks are a new model of wireless communication for mobile crowds. No immobile structure such as base locations as mobile switching nodes [9] within each other radio range interconnect directly via wireless links while these which are distant apart rely on other nodes to dispatch messages. Node mobility causes frequent changes in topology. Since there is no administrative facility and central entity in a mobile ad hoc network, the nodes need to collaboratively support all the network activities. To provide reliable communication service under adversarial surroundings, securing the elementary network process suits one of the primary concerns in WANETs [9]. This section describes the main types of misbehavior that can be formed in an ad hoc network.

\section{B. Challenges}

Use of wireless links reduces an Ad-hoc network vulnerable to link attacks ranging from passive eavesdropping to active impersonation, message reiteration and message alteration. Eavesdropping power give an attacker access to secret information thus violating privacy. Active attacks could range from deleting messages, injecting mistaken messages; copy a node etc thus violating accessibility, integrity, certification and non-repudiation. In a hostile environment nodes can be roaming freely with comparatively poor physical protection have non-negligible probability has been compromised. Therefore, there is a purpose to consider malicious attacks not only from outside but also from within the network from conciliated nodes. For high survivability ad-hoc networks should have a distributed architecture with no central entities, centrality increases vulnerability. Ad-hoc network is changing network due to frequent accommodate with topology easily. Even the trust relationships among individual nodes also changes, especially when some nodes are found to be compromised. Security mechanism necessary feature to be fly (dynamic) and not static and should be scalable.

\section{Related Work}

In several solicitations [10], such as disseminating stock quotes and video conferencing, The data origin authentication of requires the acknowledged traffic in several solicitations [10] such as disseminating stock quotes and video conferencing. Therefore, data origin authentication is a significant element in the multicast security architecture. In this paper says about the analysis and category of modern works trading with 
the data origin authentication problem in group communication. The discussion and comparison is based appropriate performance criteria.

Multicast stream authentication and signing [1] is a major criterion. The main encounters are four folded. First, authenticity must be assured even only the sender of the data alone is trusted. Second, the scheme needs to scale potentially millions of receivers. Third, flowed media distribution can have heavy packet loss. Finally, the system longings to be efficient to support fast packet rates. Here we proposed couple of efficient schemes, TESLA and EMSS, for secure lossy multicast streams. TESLA, short for Timed Efficient Stream Loss-tolerant Authentication is used for the symmetric cryptographic primitives such as pseudorandom functions (PRFs) and message authentication codes (MACs). It is based on timed release of keys done by the sender. EMSS, short for Efficient Multi-chained Stream Signature is based on signing a small number of special packets in a data stream. Each packet is related to a signed packet via multiple hash chains.

The $\mathrm{BiBa}$ signature scheme [11], a new signature construction that uses one-way functions without trapdoors. The features of this signature are low verification overhead and a relatively small signature size. BiBa has smaller signatures and is at least twice as fast to verify. One of the main challenges of securing broadcast communiqué is source authentication, which permits all receivers to verify the origin of the data. A new construction based on this signature, that achieves all our desired properties, such as the sender to generate the substantiation information, and that it requires movable time synchronization between the sender and receivers. On the downside, the public key is larger and the signature generation overhead is higher than for previous approaches.

A main challenge is to judge [2] whether or not a routing message originates from a trustworthy node. This article surveys the state of the art within key management for ad hoc networks, and investigates their applicability for network-layer security. The investigation puts some emphasis on their applicability in scenarios such as emergency and rescue operations, as this work was originated by a study of security in WANETs for emergency and rescue operations. The difficulty is optimal combination of bandwidth efficiency and robustness against link loss under a given power consumption. Also, secure and efficient key revocation remains an open challenge in WANETs

Security in wireless ad hoc networks [5] is hard to achieve due to the vulnerability of links, limited physical protection, and the absence of a centralized management point. In this paper a distributed public key authentication service to protect the network containing malicious and conniving nodes. Their solution was built on a clustering based network model and a trust model. These models allow portable hosts to monitor and rate each other with an authentication metric. Some issues including the success rate, failed rate, unreachable rate, and false-positive and false-negative error rates are difficult to evaluate accurately.

\section{A. Architecture model}

\section{System Model}

An ad-hoc network is a collection of autonomous nodes that together set up a topology model. Communications among nodes are via multi-hop routes. The nodes are grouped into clusters and they established securely by using pre-distributed public keys or applying identity based asymmetric key-pair cryptographic methods. Each cluster is controlled by a cluster-head, which is reachable to all nodes in its cluster. Nodes that have links to peers in other clusters would serve as gateways. The presence of gateways between two clusters implies that the heads of these clusters are $r$ each able to each other over multi-hop path and that these two clusters are considered neighbors.

It is based on the scheme of TESLA [1]. If a node moves out its current cluster and joins another, it is assumed that the associated cluster heads will conduct a handoff to update each other about the change in membership of their clusters; other cluster-heads will not be involved in the handoff events outside their clusters.

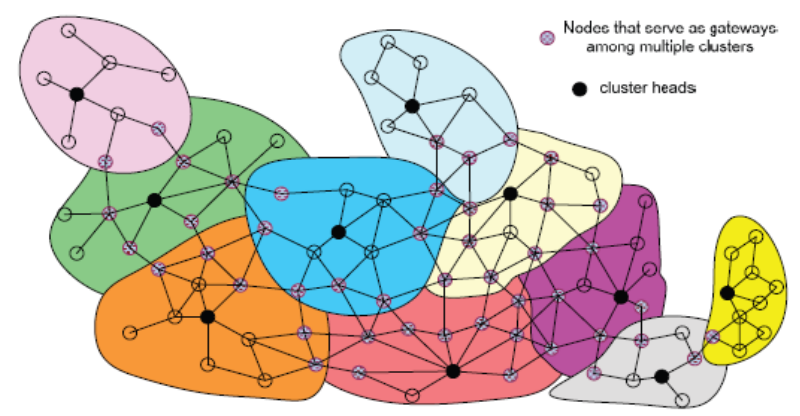

Fig.1. An example clustered ad-hoc network where each node is reachable to its cluster head via at most 1-hop (2-hop clustering). Nodes that have links to other clusters serve as gateways. 


\section{B. Key Management}

Cryptographic systems such as digital signatures are often engaged to protect both routing info also data. Public key schemes are generally adopted because of its greater hand in key distribution. In public key arrangement each node has a public/private key pair. Public keys are disseminated to another nodes, while private keys are conserved to nodes themselves and that too confidentially. Third party (trusted) called Certification Authority (CA) is used for key administration.CA has a public/private key pair, the public key known to every node and signs certificates binding public keys to nodes. The trusted CA has to abide online to reproduce the current bindings, since the bindings could accommodate overtime. Public key should be taken back if the owner node is no longer trusted or is out of network.

A single key administration service for an ad-hoc network is perhaps not a good idea, since it's likely to become Achilles' repair of the network. If CA is down/absent nodes cannot get the current public keys of other nodes to establish secure connection. Also if a CA is negotiated, the attacker can sign any flawed certificates with the private key. Naive duplication of CA can make the network more vulnerable, since exposing of a single duplication can cause the system to fail. Hence it's more sensible to distribute the trust to a set of nodes by letting these nodes share the key organization responsibility.

\section{Overall Model with integrity checking}

Tired authentication is possible because of multicast protocol. MAC is used for the authentication purpose of data and source integrity. We generate a new network topology model called Central Authority (CA) to keep track of all the receivers in the Ad-Hoc network. The keys are necessary to authenticate the messages between the users. The ciphered file is transferred by the sender and received by CA. Whenever CA acknowledged the file then it distribute the file to all the nodes in the Ad-Hoc network.

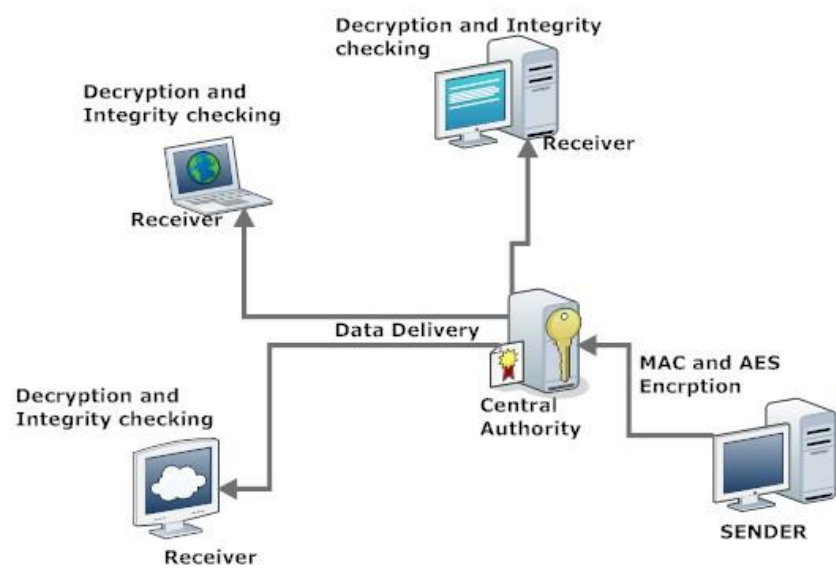

Fig.2 Overall trust model for the Central Authority

When a sender A requests to send a file to the same cluster it can proceed with one- way hash function and it generates the asymmetric key. When the same sender A requests to send a file with the cross cluster then it should proceed with Message Authentication Code (MAC) and it generates an asymmetric key with asymmetric time.

Consider a sender says A request to send a file to receiver say B in a cross cluster then it engenders MAC key with AES encryption technique. The Central Authority receives those files and it distributes to the receiver B situated in another cluster. The receiver whoever leads to receive the file should be registered earlier, then only it can able to receive the file. Now receiver B obtains the file by registered into the CA and gets the file. Once receiver collects the file formerly decrypt the file by using secret MAC key. The Integrity Checking will take place over there.

\section{Authentication Over Multicast Region}

Trilateral Conventions uses clustering to partition a network, and then authenticates multicast traffic by employing time asymmetry for similar-cluster traffic and secret information asymmetry for cross-cluster traffic. Several studies have shown that the gains achieved by clustering supersede the overheard in forming and maintain the clusters [6].

\section{A. Similar Cluster Authentication}

Grouping nodes into clusters enables having a reasonably tight bound on the end-to-end delay of packet delivery and will thus enable the use of a time asymmetry based authentication scheme. Similar-cluster 
authentication is based on TESLA [1]. Inter-cluster multicast traffic will be authenticated differently as explained below. A source node generates a chain of one-time-use keys using the hash function, e.g., MD5, SHA-1, etc., and shares only that last generated key with receivers. A message can be legitimated only when the used key in the chain is revealed. The approach has two distinctive advantages, namely:

- Basically due to a small MAC overhead, a single MAC is used per every multicast packet for all receivers.

- A missed key is always considered as a lost packet and it would not obstruct the authentication process since a receiver can refer back the previous key.

The size of the time interval was determined whenever the key is revealed, depends on the clock jitter among each cluster nodes in the cluster and on the maximum end-to-end delay between a sender and receivers. Uncertainty about these factors causes the source to be extra conservative in revealing the keys and it thus slows down the data transmission rate.

In this paper, the concern about the authentication delay is generally addressed by the fact that the cluster includes just a subset of the network nodes. The maximum end-to-end delay experienced by an intra-cluster multicast will be mostly dependent on the cluster radius. By controlling the radius of the cluster at the time of cluster formation, i.e., deciding the distance in terms of the number of hops between a member node and the cluster-head [9], [4], it will be possible to tackle this issue. Furthermore, clustering will make it more feasible to synchronize the clock of the nodes in the cluster with some reasonable accuracy. It is well known that for distributed clock synchronization schemes the accuracy diminishes with increased node population [8].

\section{B. Cross-Cluster Authentication}

Authentication based on time asymmetry requires clock synchronization and thus does not suit large networks. For similar-cluster multicast traffic, TAM applies a strategy based on secret information asymmetry and engages the cluster heads in the authentication process. Basically, the source " $s$ " that belongs to Cluster $i$ will send the multicast packets to the heads of all clusters that have designated receivers. The rationale is that the MAC will be associated with the cluster rather than the nodes and thus the overhead is reduced expressively. In other words, the multicast from source s consists of multiple multicasts; (1) from $s$ to all relevant cluster heads, (2) a dissimilar multicast within each of the target clusters to relay the message to designated receivers. This can also be advantageous if node mobility is to be dealt with. A node that switches from one cluster to another would only introduce local changes and would not require special handling by the source with respect to the authentication process. The process goes as follows. The source will generate a group of $M$ keys. Each of the $N C L$ clusters in the network will be assigned a share $L$ of keys, with $M<L \times N C L$.

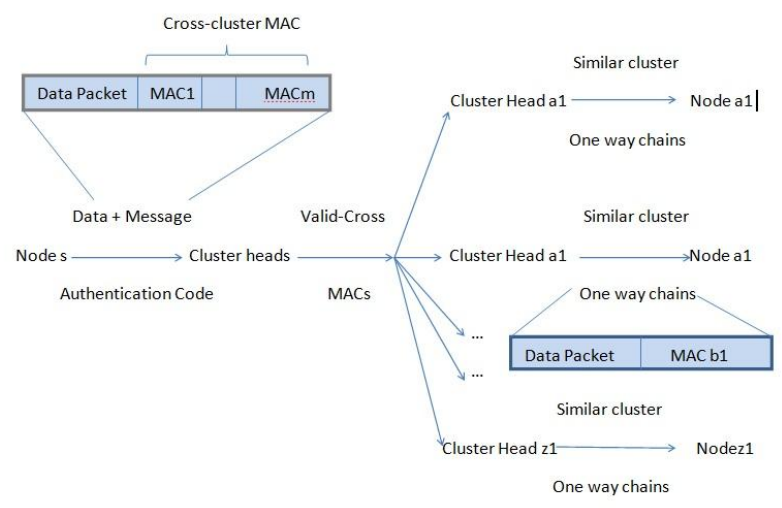

Fig-3 : Illustrates packet transferring of cross and similar cluster over network.

The key share will be sent securely, e.g. using asymmetric cryptology basis protocol, to the heads of the individual clusters. The source will then append multiple MACs to the multicast packet; each MAC is based on a distinct key.

\section{Result Analysis}

This section describes the result analysis based on the performance and evaluation analysis. In the experiments, compared the existing and proposed system of performance evaluation. The results of the individual experiments are averaged over 30 runs using distinct network topologies. In conclusion, the performance favors fewer clusters count, and dense and highly connected clusters.

The main advantage over this proposed method is occupying of memory by the central authority is lesser than the previous existing method. The efficiency is also higher while using central authority. 


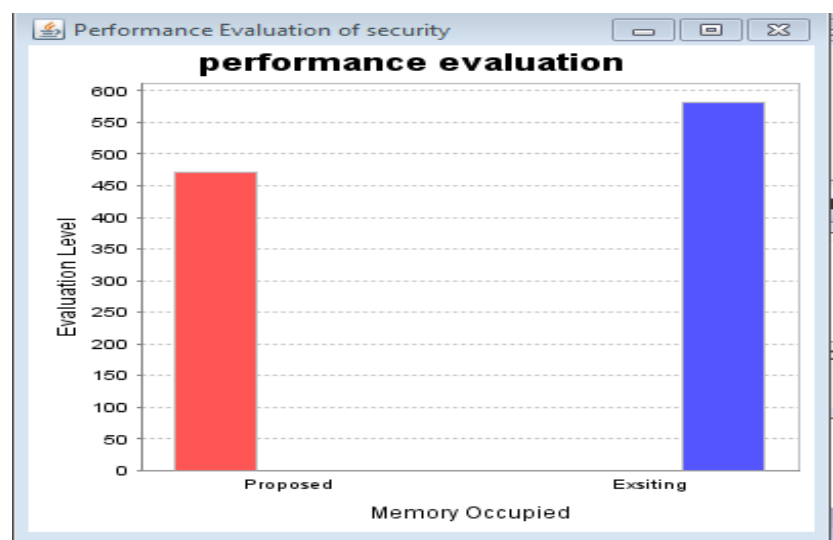

(a)

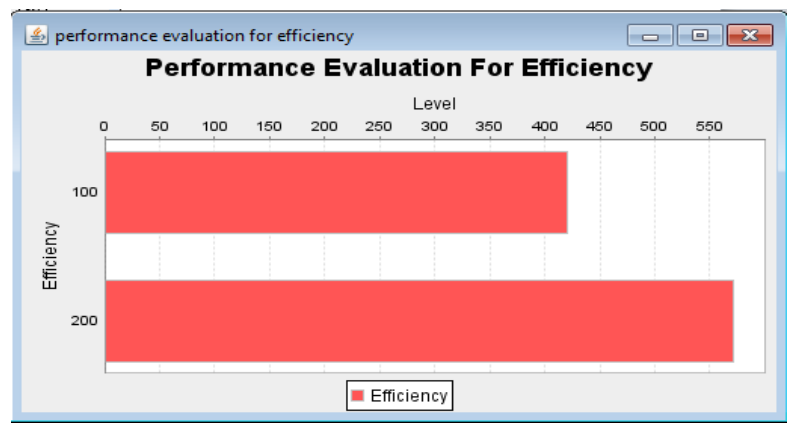

(b)

Fig- 4(a), 4(b) The results are variation of memory occupied and performance evaluation of the multicast packet to all receivers.

\section{Conclusion}

In recent years there has been a growing interest in the use of ad-hoc networks in security-sensitive applications such as digital battlefield, situation awareness, and border protection. The collaborative nature of these applications makes multicast traffic very common. Securing such traffic is of excessive importance, mainly authenticating the source and message to prevent any infiltration attempts by an intruder. Contemporary source authentication schemes found in the literature either introduce excessive overhead or do not scale for large networks. This paper has presents which combines both time and secret-information asymmetry in order to achieve scalability and resource efficiency. The key sharing through a common central authority and trilateral conventions used in this paper reduced the overhead of MAC. Our future work plan includes the study of enhanced guarantee for key sharing.

\section{References}

[1] Perrig, R. Canetti, D. Song, and D.Tygar, "Efficient authentication and signing of multicast streams over lossy channels," in Proc. 2000 IEEE Symposium Security Privacy.

[2] A.M. Hegland, E. Winjum, S. F. Mjolsnes, C. Rong, O. Kure, and P. Spilling, "A survey of key management in ad hoc networks," IEEE Commun. Surveys \& Tutorials, vol. 8, no. 3, pp. 48-66, Dec. 2006.

[3] R. Safavi-Naini and H. Wang, "Multi-receiver authentication codes: models, bounds, constructions, and extensions," Inf. Computation, vol. 151, no. 1-2, pp. 148-172, May 1999.

[4] J. Y. Yu and P. H. J. Chong, "A survey of clustering schemes for mobile ad hoc networks," IEEE Commun. Surveys \& Tutorials, vol. 1 , no. 1, pp. 31-48, 2005.

[5] P. B. Velloso, et al., "Trust management in mobile ad hoc networks using a scalable maturity-based model," IEEE Trans. Network Service Management, vol. 7, no. 3, Sep. 2010.

[6] L. Junhai, Y. Danxia, X. Liu, and F. Mingyu, "A survey of multicast routing protocols for mobile ad-hoc networks," IEEE Commun. Surveys \& Tutorials, vol. 11, no. 1, pp. 78-91, first quarter 2009.

[7] M. Younis, O. Farrag, and S. Lee, "Cluster mesh based multicast routing in MANET: an analytical study," in Proc. 2011 IEEE International Conf. Commun..

[8] K. Marzullo and S. Owicki, "Maintaining the time in a distributed system," in Proc. 1983 ACM Symposium Principles Distrib. Computing.

[9] R. Canetti et al., "Multicast security: a taxonomy and efficient constructions," in Proc. 1999 IEEE INFOCOM.

[10] Y. Challal, H. Bettahar, and A. Bouabdallah, "A taxonomy of multicast data origin authentication, issues and solutions," IEEE Commun. Surveys \& Tutorials, vol. 6, no. 3, pp. 34-57, 2004.

[11] Perrig, "The BiBa one-time signature and broadcast authentication protocol," in Proc. 2001

[12] Mohamed Younis, Osama Farrag, Bryan Althouse "TAM: Tiered Authentication of Multicast Protocol in Ad-hoc Networks" in IEEE Transactions On Network And Service Management, Vol. 9, No. 1, March 2012 